\title{
LOCATION PROBLEMS IN TRANSPORTATION NETWORKS
}

It is known that many optimisation problems on networks are NP-hard. However, it seems that the real transport networks have some interesting properties which allow us to find a ,good“ solution in reasonable time. In this paper, we suggest and study some new parameters of the transportation networks which could be useful in optimisation problems. We define the evenness and the robustness of the solution. We also concern ourselves with the statistical distribution of distances and edge values in transportation networks.

Keywords: Transportation networks, Euclidean networks, robustness, distribution of distances.

\section{Introduction and preliminaries}

It is well known fact that many optimisation algorithms work more successfully in real networks than in random graphs. For example, the greedy method for the traveling salesman problem gives, in real transportation networks, better results than in general graphs [1]. The difference is caused by triangle inequality which usually holds in transportation networks. We can also mention networks with Euclidean metric. Their properties allow us to suggest more efficient algorithms [2].

We will need the following definitions from [3 and 4]. Network is an ordered quadruple $G=(V, E, c, w)$, where $V \neq \emptyset$ is the set of vertices, $E \neq \emptyset$ is the set of edges (oriented or un-oriented), $c: E \rightarrow R_{0}^{+}$is a function which represents the length of edges and $w: V \rightarrow N_{0}$ represents the weights of vertices. Let $d_{G}(u, v)$ be the distance between vertices $u$ and $v$ in network $G$ and let $d_{G}(v, D)=\min \left\{d_{G}(v, u) \mid u \in D\right\}$ be the distance between a set $D \subset V$ and a vertex $v$ in network $G$.

The eccentricity of a set $D$ is $e c_{G}(D)=\max \left\{d_{G}(v, D) \mid v \in V\right\}$.

The weighted eccentricity of $D$ is $\operatorname{ecc}_{G}(D)=\max \left\{w(v) \cdot d_{G}(v, D) \mid v \in V\right\}$.

The total distance of vertices of $G$ from $D$ is $f_{G}(D) \sum_{v \in V} d_{G}(v, D)$

The total weighted distance of vertices of $G$ from $D$ is $f_{G}^{w}(D)=\sum w(v) \cdot d_{G}(v, D)$.
Now we are able to define various location problems on network $G$.

Set $D \subset V$ is the $p$-center of $G$, if $|D|=p$ and $e c_{G}(D) \leq e c_{G}\left(D^{\prime}\right)$ for any $p$-element subset $D^{\prime} \subset V$.

Set $D \subset V$ is the weighted $p$-center of $G$, if $|D|=p$ and $\operatorname{ecc}_{G}(D) \leq \operatorname{ecc}_{G}\left(D^{\prime}\right)$ for any $p$-element subset $D^{\prime} \subset V$.

Set $D \subset V$ is the $p$-median of $G$, if $|D|=p$ and $f_{G}(D) \leq f_{G}\left(D^{\prime}\right)$ for any $p$-element subset $D^{\prime} \subset V$.

Set $D \subset V$ is the weighted $p$-median of $G$, if $|D|=p$ and $f_{G}(D) \leq f_{G}\left(D^{\prime}\right)$ for any $p$-element subset $D^{\prime} \subset V$.

Set $D \subset V$ is the anti-p-center of $G$, if $|D|=p$ and $e c_{G}(D) \leq e c_{G}\left(D^{\prime}\right)$ or any $p$-element subset $D^{\prime} \subset V$.

Set $D \subset V$ is the $p$-maxian of $G$, if $|D|=p$ and $f_{G}(D) \leq f_{G}\left(D^{\prime}\right)$ for any p-element subset $D^{\prime} \subset V$.

Definitions of weighted anti- $p$-center and weighted $p$-maxian are similar. It is known that the mentioned problems are $N P$-hard (if $p$ is a part of the input) [5 and 6]. This is the reason why heuristic algorithms are usually used for finding a suboptimal solution [7, 8 and [9]. The only exception is the anti-p-center problem, which is polynomial - as it was shown in [10].

\section{Robustness of a solution}

In this section we introduce the robustness of a solution of various location problems. Let $D \subset V$ (where $D \mid=p$ ) be a solution (not necessarily optimal) of a given location problem with objective function $\min h_{G}$ (where $h_{G} \in\left\{e c_{G}, e c c_{G}, f_{G}, f_{G}^{w}\right\}$ ). We can suppose that $k$ edges of $G$ are not rideable. We denote the set of these edges by $F$. It is reasonable to investigate the changes of objective function in network $G-K$, where $G-F$

\footnotetext{
* Peter Czimmermann

Department of Mathematical Methods and Operations Research, Faculty of Management Science and Informatics, University of Zilina, Slovakia E-mail: peter.czimmermann@fri.uniza.sk
} 
denotes the network obtained from $G$ by deleting the edges from $F$. The $k$-robustness of set $D$ for a given objective function $h_{G}$ is $\min =\left\{h_{G}(D) / h_{G-F}(D)|F \subset E| E \mid,=k\right\}$.

The problem of finding a $k$-robustness of $D$ for function $e c_{G}(D)$ seems to be similar to the problem of edge $k$-connectivity in graphs which is solvable in polynomial time [11]. An exact polynomial algorithm or heuristic based on edge $k$-connectivity with tests on real data will be published in the next paper. The application of $k$-robustness is in facility location problems. For example, we need to locate $p$ emergency medical stations. We have solutions $D_{1}, D_{2}, \ldots, D_{a}$ of similar quality. Then the robustness can be the next criteria for choosing one solution, because it says more about stability of the solution when there are parts of the network that are not rideable.

Similar concepts are studied for example in [12], where the authors deal with a facility (vertex) disruption, and [13], where the author studies the network reliability in general.

\section{Evenness of a solution}

In [2], the Euclidean metric, the triangle inequality and its impact on the solution of the $p$-median problem have been studied. It follows from the results that practical algorithms are more efficient in networks with these properties. We would like to continue in the research started in the above mentioned work. Hence, we suggest the study of three new parameters of solutions of various location problem (two of them are taken over from mathematical statistics). We would like to compare these parameters in networks and in graphs without the above mentioned properties. These parameters are the mean of vertex distances, variance and evenness. Let the set $D \subset V(|D|=p)$ be given. The arithmetic mean of distances (of vertices) in $D$ is

$$
\bar{d}_{G}(D)=\frac{2}{p(p-1)} \cdot \sum_{\substack{u \in D \\ v \in D}} d_{G}(u, v) .
$$

The variance of distances (of vertices) in $\mathrm{D}$ is

$$
\begin{aligned}
& \operatorname{Var}(D)=\frac{2}{p(p-1)} \cdot \sum_{\substack{u \in D \\
v \in D}}\left(d_{G}(u, v)-\bar{d}_{G}(D)\right)^{2} . \\
& \text { The evenness of D in } \mathrm{G} \text { is } \\
& \mathcal{E}_{G}(D)=\operatorname{Var}(D) / \operatorname{Var}(V) .
\end{aligned}
$$

We suppose that it is possible to approximate the mean, variance and evenness of the optimal solution from the properties of networks with Euclidean metrics. If we have appropriate approximations of these values, then we can restrict the set of admissible solutions. Computation of the optimal solution could be faster than computation in general graphs. It is convenient to test the properties of optimal solutions in large networks for the anti p-center problem, since this problem is solvable in polynomial time [10].

\section{Distribution of distances}

In this section we develop some ideas from the work [2], where the authors solve the $p$-median model using standard linear programming (plus branch and bound) method and the vertex substitution heuristic. The types of networks studied in [2] include Euclidean networks (distances between points are measured by Euclidean metric), path networks (with triangle inequality) and random distance networks. It follows from the results that the above mentioned methods are more efficient in Euclidean networks and path networks (which are similar to the actual transportation networks) even if the methods were not suggested for these special types of networks. We suppose that the algorithms using specific properties of these networks could provide significantly better results.

Important properties could be the distribution of distances and the distribution of edge lengths. Our tests on the road network of Slovakia show the distribution of edge lengths which is in Fig. 1.

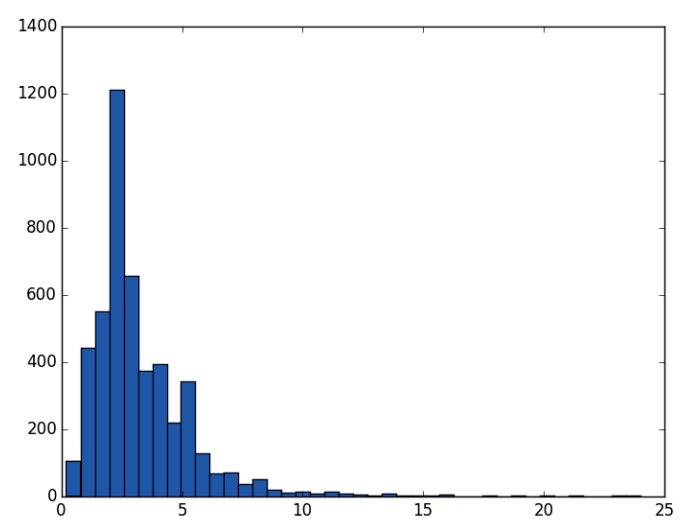

Fig. 1 The distribution of edge lengths in the road network of Slovakia

This distribution is similar to the Maxwell-Boltzmann distribution of molecule speeds in idealised gas [14] with the distribution function $f(x)=\sqrt{2 /\left(\pi a^{6}\right)} \cdot x^{2} \cdot e^{-x^{2} /\left(2 a^{2}\right)}$, where $a$ is an appropriate constant. The proof, that the distribution of edge lengths could be approximated by the Maxwell-Boltzmann distribution function, involves finding a special, continuous embedding of the road network into the three dimensional Euclidean space which preserves the incidence relation of a network and maps every edge to line segment with the same length. The existence of such embedding remains an open question. The next step of the proof involves finding the representation of the road network by the above mentioned 
physical model, where edge lengths mean speeds of the particles. We are preparing a paper which is devoted to this topic.

If we consider the distribution of distances in the road network, then we achieve a similar situation. The results for distance distribution in Euclidean networks can be found in [2] and, for distance distribution of bus stops and stations in China, can be found in [15], where the authors work with a gamma distribution. We think that the best approximation of the distance distribution can be found in [16], where the probability distribution for the distance between two random points in a rectangle with given sides is determined. Its probability density function for a rectangle with sides $a, b$ is

$f(x)=\frac{2 x}{a b}\left(-\frac{2 x}{a}-\frac{2 x}{b}+\pi+\frac{x^{2}}{a b}\right)$, for $0<x \leq a$,
$f(x)=\frac{2 x}{b}\left(-\frac{2 x}{a^{2}}-\frac{1}{b}+\frac{2}{a} \arcsin \left(\frac{a}{x}\right)+\frac{2}{a^{2}} \sqrt{x^{2}-a^{2}}\right)$

for $a<x \leq b$,

and

$f(x)=\frac{2 x}{a b}\left(2 \arcsin \left(\frac{a}{x}\right)+\frac{2}{a} \sqrt{x^{2}-a^{2}}+2 \operatorname{arcin}\left(\frac{b}{x}\right)+\right.$ $\left.+\frac{2}{b} \sqrt{x^{2}-b^{2}}\right)-\frac{2 x}{b^{2}}-\frac{2 x}{a^{2}}-\frac{2 \pi x}{a b}-\frac{2 x^{3}}{a^{2} b^{2}}$, for $b<x \leq \sqrt{a^{2}+b^{2}}$

We suppose that appropriate solutions of the $p$-center and $p$-median problem have the same distance distribution as the whole network. Our goal is the suggestion of a method for solving location problems which would be more effective on Euclidean and path networks than existing methods. Information about behaviour of solutions (distance distribution and evenness) can help us to find some new algorithms.

\section{Conclusions}

This paper is an introduction to our study of parameters and properties of transportation networks. We suppose that new knowledge in this area of research could bring more efficient methods for solving the problems that are too hard for exact computation in general.

\section{Acknowledgment}

The research was supported by the Scientific Grant Agency of the Ministry of Education of the Slovak Republic under project VEGA 1/0518/15 "Resilient rescue systems with uncertain accessibility of service".

\section{References}

[1] COOK, W. J.: In Pursuit of the Traveling Salesman: Mathematics at the Limits of Computation, Princeton University Press, 2012, ISBN 978-0691152707.

[2] SCHILling, D. A., ROSING, K. E., REVELLE, C. S.: Network Distance Characteristics that Affect Computational Effort in P-Median Location Problems, European J. of Operational Research 127, p. 525 - 536, 2000.

[3] PALUCH, S.: Graph Theory (in Slovak), EDIS: University of Zilina, p. 173 - 177, 2001.

[4] PALUCH, S., PESKO, S.: Quantitative Methods in Logistics (in Slovak), EDIS: University of Zilina, 2006.

[5] KARIV, O., HAKIMI, S. L.: An Algorithmic Approach to Network Location Problems, Part I: The p-centers, SIAM J. of Applied Mathematics, 37, p. 513 - 538, 1979.

[6] KARIV, O., HAKIMI, S. L.: An Algorithmic Approach to Network Location Problems, Part II: The p-medians, SIAM J. of Applied Mathematics, 37, p. 539 - 560, 1979.

[7] JANACEK, J., JANOSIKOVA, L., BUZNA, L.: Optimized Design of Large-Scale Social Welfare Supporting Systems on Complex Networks, Chapter 12 in Handbook of Optimization in Complex Networks: Theory and Applications, Springer Optimization and Its Applications 57, editors: Thai, M. T., Pardalos, P. M., 2012.

[8] JANACEK, J., Radial approach to the emergency public service system design with generalized system utility, International J. of Applied Mathematics and Informatics, 8: 7-14, 2014.

[9] JANOSIKOVA, L.: Emergency Medical Service Planning, Communications - Scientific Letters of the University of Zilina, 9(2): 64-68, 2007.

[10] CZIMMERMANN, P., PESKO, S.: A Polynomial Algorithm for a Particular Obnoxious Facility Location Problem, Proc. of the 13 ${ }^{\text {th }}$ Intern. Symposium on Operational Research in Slovenia, SOR'15, Bled, 2015.

[11] PLESNIK, J.: Graph Algorithms (in Slovak), VEDA: Bratislava, 1983.

[12] SCAPARRA, M., P., CHURCH, R., L.: Location Problems under Disaster Events, Chapter 24 in Location Science, Springer, editors: Laporte, G., Nickel, S., Saldanha da Gama, F., 2015. 
[13] SHOOMAN, M., L.: Reliability of Computer Systems and Networks: Fault Tolerance, Analysis, and Design, John Wiley \& Sons : New York, 2002.

[14] KREMPASKY, J., Physics (in Slovak), ALFA, Bratislava, 1988

[15] LU, H., SHI, Y.: Complexity of Public Transport Networks, Tsinghua Science and Technology, vol. 12, No. 2, 2007.

[16] PHILIP, J.: The Probability Distribution of the Distance between two Random Points in a Box, Trita Mat, 7(10), 2007. 